\title{
The transcriptional response of Arabidopsis leaves to Fe deficiency
}

\section{Jorge Rodríguez-Celma ${ }^{1}$, I Chun Pan ${ }^{1}$, Wenfeng Li ${ }^{1}$, Ping Lan ${ }^{1}$, Thomas J. Buckhout ${ }^{2}$ and Wolfgang Schmidt ${ }^{1,3,4 *}$}

1 Academia Sinica, Institute of Plant and Microbial Biology, Taipei, Taiwan

2 Institute of Biology, Humboldt University Berlin, Berlin, Germany

${ }^{3}$ Biotechnology Center, National Chung-Hsing University, Taichung, Taiwan

${ }^{4}$ Genome and Systems Biology Degree Program, College of Life Science, National Taiwan University, Taipei, Taiwan

\section{Edited by:}

Jean-Francois Briat, Centre National de la Recherche Scientifique, France

\section{Reviewed by:}

Petra Bauer, Saarland University, Germany

Naoko K. Nishizawa, The University of Tokyo, Japan

*Correspondence:

Wolfgang Schmidt, Academia Sinica Institute of Plant and Microbial

Biology, Academia Road 128,

Taipei 11529, Taiwan

e-mail:wosh@gate.sinica.edu.tw
Due to its ease to donate or accept electrons, iron ( $\mathrm{Fe}$ ) plays a crucial role in respiration and metabolism, including tetrapyrrole synthesis, in virtually all organisms. In plants, $\mathrm{Fe}$ is a component of the photosystems and thus essential for photosynthesis. Fe deficiency compromises chlorophyll (Chl) synthesis, leading to interveinal chlorosis in developing leaves and decreased photosynthetic activity. To gain insights into the responses of photosynthetically active cells to Fe deficiency, we conducted transcriptional profiling experiments on leaves from Fe-sufficient and Fe-deficient plants using the RNA-seq technology. As anticipated, genes associated with photosynthesis and tetrapyrrole metabolism were dramatically down-regulated by Fe deficiency. A sophisticated response comprising the down-regulation of HEMA1 and NYC1, which catalyze the first committed step in tetrapyrrole biosynthesis and the conversion of $\mathrm{Chl} b$ to $\mathrm{Chl} a$ at the commencement of $\mathrm{Chl}$ breakdown, respectively, and the up-regulation of CGLD27, which is conserved in plastid-containing organisms and putatively involved in xanthophyll biosynthesis, indicates a carefully orchestrated balance of potentially toxic tetrapyrrole intermediates and functional end products to avoid photo-oxidative damage. Comparing the responses to Fe deficiency in leaves to that in roots confirmed subgroup $1 \mathrm{~b}$ bHLH transcription factors and POPEYE/BRUTUS as important regulators of Fe homeostasis in both leaf and root cells, and indicated six novel players with putative roles in $\mathrm{Fe}$ homeostasis that were highly expressed in leaves and roots and greatly induced by Fe deficiency. The data further revealed down-regulation of organ-specific subsets of genes encoding ribosomal proteins, which may be indicative of a change in ribosomal composition that could bias translation. It is concluded that Fe deficiency causes a massive reorganization of plastid activity, which is adjusting leaf function to the availability of Fe.

Keywords: Fe deficiency, chlorophyll metabolism, ribosomes, Fe homeostasis, reactive oxygen species, RNA-seq

\section{INTRODUCTION}

In leaves, the vast majority of $\mathrm{Fe}$ is associated with the chloroplast, serving as a cofactor in all three photosynthetic electron transfer complexes. Iron deficiency is manifested in the interveinal chlorosis of developing leaves, which has been described more than one-hundred years ago as a diagnostic symptom of Fe deficiency (Gris, 1844). Chlorosis is caused by compromised chloroplast development and impaired chlorophyll (Chl) biosynthesis and is associated with dramatically decreased photosynthetic rates (Terry, 1980). The first step in tetrapyrrole biosynthesis is the formation of 5-aminolevulinic acid (ALA). Synthesis of ALA is regarded as the rate-limiting step in tetrapyrrole synthesis, and the glutamyl-tRNA reductase is the point of regulation (Tanaka et al., 2011). Interrupting the Chl biosynthesis pathway is detrimental to the plant due to severe oxidative damage caused by the generation of singlet oxygen by free tetrapyrroles upon illumination (op den Camp et al., 2003). A sensitive Fe sensing system associated with chloroplast function would allow plants to anticipate the inability to complete tetrapyrrole synthesis, to shut off the first committed step in the pathway and thus to avoid potentially detrimental consequences.

How $\mathrm{Fe}$ is sensed by plants is not known. In Arabidopsis roots, the earliest hub of the Fe signaling cascade is the bHLH protein FER-LIKE IRON DEFICIENCY INDUCED TRANSCRIPTION FACTOR (FIT), which positively controls a set of genes that are involved in the acquisition and distribution of Fe. Among them are the key genes that mediate rhiszosphere acidification (AHA2; Santi and Schmidt, 2009), the solubilization of un-available Fe pools (F6'H1 and PDR9; Rodríguez-Celma et al., 2013), the reduction of $\mathrm{Fe}(\mathrm{III})$ chelates (FRO2; Robinson et al., 1999) and the uptake of the resulting $\mathrm{Fe}(\mathrm{II})$ (IRT1; Eide et al., 1996; Ling et al., 2002; Colangelo and Guerinot, 2004; Jakoby et al., 2004). FIT forms heterodimers with four Fe-responsive bHLH proteins, bHLH38, bHLH39, bHLH100, and bHLH101 (Yuan et al., 2005, 2008; Wang et al., 2012), presumably controlling different subsets of genes. Another bHLH transcription factor, POPEYE (PYE), 
negatively regulates a non-overlapping set of genes involved in Fe mobilization in roots and translocation of Fe to above-ground plant parts in association with the bHLH protein IAA-LEUCINE RESISTANT3 (ILR3/bHLH105) (Long et al., 2010). ILR3 also interacts with the putative DNA-binding E3 ubiquitin-protein ligase BRUTUS (BTS), which positively regulates the same set of genes. This dual regulation is thought to allow a fine-tuning of the expression of genes involved in Fe acquisition and cellular Fe homeostasis (Long et al., 2010).

Little information is available on putative candidates involved in sensing and signaling of Fe in chloroplasts or leaves. Sensing of the Fe status in the plastid would not only allow a close coupling of Chl synthesis, chloroplast development and photosynthesis rate, thus optimizing plant performance, but would also enable the plants to communicate the Fe status of the chloroplast to roots and to adjust Fe uptake to demand. Furthermore, the mechanisms that underlie Fe uptake in leaves have not been thoroughly described. Fe is transported to leaves as $\mathrm{Fe}^{3+}$-citrate chelate (Rellán-Álvarez et al., 2010), and might then be reduced either enzymatically by members of the FRO family or nonenzymatically by ascorbate. Based on their expression patterns and chlorotic mutant phenotypes, the YELLOW STRIPE-LIKE (YSL) transporters YSL1, YSL2, and YSL3 have been implicated in the uptake of Fe that has arrived in leaves via xylem transport (Didonato et al., 2004; Waters et al., 2006). Land plants evolved from chlorophyte algae, suggesting that conserved responses to $\mathrm{Fe}$ deficiency should be present in both groups. Indeed, a recent survey on transcriptional responses of Chlamydomonas reinhardtii revealed an overlap with Fe-responsive genes of Arabidopsis thaliana, including the metal transporters IRT1, IRT2, NRAMP4, and the P-type $\mathrm{H}^{+}$-ATPase AHA2 (Urzica et al., 2012). However, the development of multicellularity in plants is accompanied by functional diversity of cells, tissues and organs. The contrasting roles of leaves and roots as sinks and sources for Fe are indicative of fundamentally different transcriptional responses in these organs.

To dissect the Fe deficiency response of leaf cells, we analyzed the changes in the transcriptome upon exposure to Fe deficiency in Arabidopsis leaves and compared this response with a previously published RNA-seq data set collected following the same treatment. The analysis revealed a suite of genes functioning in photosynthesis, chloroplast development and Chl synthesis that was rapidly and robustly regulated by $\mathrm{Fe}$ in leaves, indicating that the control of plastid function is a conserved and integral component of the Arabidopsis response to Fe deficiency. Our analysis further suggests that the composition of ribosomes by $\mathrm{Fe}$ is affected in an organ-specific manner, probably biasing translation. Lastly, we show that a small subset of highly expressed genes is robustly regulated by Fe deficiency in both roots and leaves, suggesting putative roles of the encoded proteins in Fe metabolism and the regulation of Fe homeostasis.

\section{MATERIALS AND METHODS PLANT GROWTH CONDITIONS}

Arabidopsis thaliana plants were grown in a growth chamber on an agar-based medium as described by Estelle and Somerville (1987). Seeds of the accession Columbia (Col-0) were obtained from the Arabidopsis Biological Resource Center (Ohio State University). Seeds were surface-sterilized by immersing them in $5 \%(\mathrm{v} / \mathrm{v}) \mathrm{NaOCl}$ for $5 \mathrm{~min}$ and $70 \%$ ethanol for $7 \mathrm{~min}$, followed by four rinses in sterile water. Seeds were placed onto Petri dishes and kept for $1 \mathrm{~d}$ at $4^{\circ} \mathrm{C}$ in the dark, before the plates were transferred to a growth chamber and grown at $21^{\circ} \mathrm{C}$ under continuous illumination ( $50 \mu \mathrm{mol} \mathrm{m}^{-2} \mathrm{~s}^{-1}$; Phillips TL lamps). The medium was composed of $(\mathrm{mM}): \mathrm{KNO}_{3}(5), \mathrm{MgSO}_{4}(2), \mathrm{Ca}\left(\mathrm{NO}_{3}\right)_{2}(2)$, $\mathrm{KH}_{2} \mathrm{PO}_{4}(2.5)$ and $(\mu \mathrm{M}): \mathrm{H}_{3} \mathrm{BO}_{3}(70), \mathrm{MnCl}_{2}(14), \mathrm{ZnSO}_{4}(1)$, $\mathrm{CuSO}_{4}$ (0.5), $\mathrm{NaCl}$ (10), $\mathrm{Na}_{2} \mathrm{MoO}_{4}$ (0.2), FeEDTA (40), 4.7 mM MES, supplemented with sucrose $(43 \mathrm{mM})$ and solidified with $0.4 \%$ Gelrite pure (Kelco). The $\mathrm{pH}$ was adjusted to 5.5 .

For RNA-seq analysis, plants were pre-cultivated for $10 \mathrm{~d}$ in a complete medium, and then transferred to fresh agar medium either with $40 \mu \mathrm{M} \mathrm{Fe}(\mathrm{III})-\mathrm{EDTA}$ (+Fe plants) or without Fe and with $100 \mu \mathrm{M}$ 3-(2-pyridyl)-5,6-diphenyl-1,2,4-triazine sulfonate (ferrozine; $-\mathrm{Fe}$ plants) to trap residual Fe. Plants were grown for $3 \mathrm{~d}$ on Fe-free media before analysis. Three independent experiments were conducted for each growth type. For RT-PCR analysis, 10-day-old plants were transferred to hydroponic solution (Buckhout et al., 2009) for $3 \mathrm{~d}$ and then transferred to Fe-free solution for 15,30 , and $45 \mathrm{~min}$, or for $1,2,4$, and $6 \mathrm{~h}$.

\section{RNA-SEO}

For RNA-seq, total RNA was extracted from the leaves and roots using the RNeasy Plant Mini Kit (Qiagen), following the manufacturer's instructions. For analysis, equal amounts of total RNA were collected and cDNA libraries for sequencing were prepared from total RNA following the manufacturer's protocol (Illumina). The cDNA libraries were subsequently enriched by PCR amplification. The resulting cDNA libraries were subjected to sequencing on a single lane of an Illumina Genome Analyzer II. RNA-seq and data collection was done following the protocol of Mortazavi et al. (2008). The length of the cDNA library varied from 250 to $300 \mathrm{bp}$ with a $5^{\prime}$-adapter of $20 \mathrm{bp}$ and a $3^{\prime}$-adapter of $33 \mathrm{bp}$ at both ends.

To quantify gene expression levels, 75-mers sequences were aligned to the genomic sequence annotated in TAIR10 using the BLAT program (Kent, 2002), and RPKM (Reads Per Kilobase of exon model per Million mapped reads) values were computed using RACKJ (Read Analysis \& Comparison Kit in Java, http://rackj.sourceforge.net/) software. Only those genes whose expression level in RPKM was over the square root of the mean expression value of the whole dataset ( $\sim 4.5 \mathrm{RPKM})$ were considered as relevant for further analyses. Differentially expressed genes were selected based on Student's $t$-test $(P<0.05)$ and delta RPKM changes bigger than the mean expression value of the whole dataset ( $\sim 20 \mathrm{RPKM})$ and/or 2-fold change in expression level between treatments.

\section{BIOINFORMATICS}

For gene clustering, we used the MACCU software (http:// maccu.sourceforge.net/) to build co-expression networks based on co-expression relationships with a Pearson's coefficient greater than or equal to 0.60 . In order to capture the tissuespecific co-expression relationships, Pearson's coefficients were computed based on robust multi-array averaged array data derived from leaf- and root-specific experiments for each tissue 
downloaded from NASCArrays (http://affymetrix.arabidopsis. info/). Visualization of the networks was performed with the Cytoscape software version 2.8.2 (http://www.cytoscape.org/).

\section{RT-PCR}

For RT-PCR, total RNA was extracted from the leaves using Trizol (Invitrogen) and DNase treated with the Turbo DNAfree Kit (Ambion) following the manufacturer's instructions. cDNA was synthesized using QuantiTect Reverse Transcription Kit (Qiagen) following the manufacturer's instructions. Real-time PCR was performed using Power SYBR Green PCR Master Mix (Applied Biosystems) on an Applied Biosystems 7500 Fast RealTime PCR System with programs recommended by the manufacturer. Samples were normalized first to an endogenous reference $(A t E F 1 \alpha)$ and then the relative target gene was determined by performing a comparative $\Delta \Delta \mathrm{Ct}$. The following primers were used:

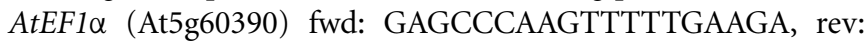
CTAACAGCGAAACGTCCCA, and HEMA1 (At1g58290) fwd: GATCTCCTTCTTCCACATCGCAA, rev: CCGCCATTGAAAC CCAAAATC.

\section{RESULTS}

To dissect processes that are triggered by decreased Fe supply, we analyzed the transcriptome of Fe-sufficient and Fe-deficient leaves using the RNA-seq technology. A total of approximately 60 million reads was collected from three independent sequencing runs per growth type and aligned to the TAIR10 annotation of the Arabidopsis genome. A set of 413 genes was differentially expressed between Fe-deficient and Fe-sufficient plants ( $t$-test $P<0.05$ with either a FC $>2$ or a $\triangle \mathrm{RPKM}>20.6)$, out of a total of 24841 genes detected in leaves from Fe-sufficient and Fe-deficient plants (Figure 1).

As anticipated from the high Fe demand of the photosynthetic electron chain, the strongest regulation was observed for photosynthesis-related genes. The expression of the PSI

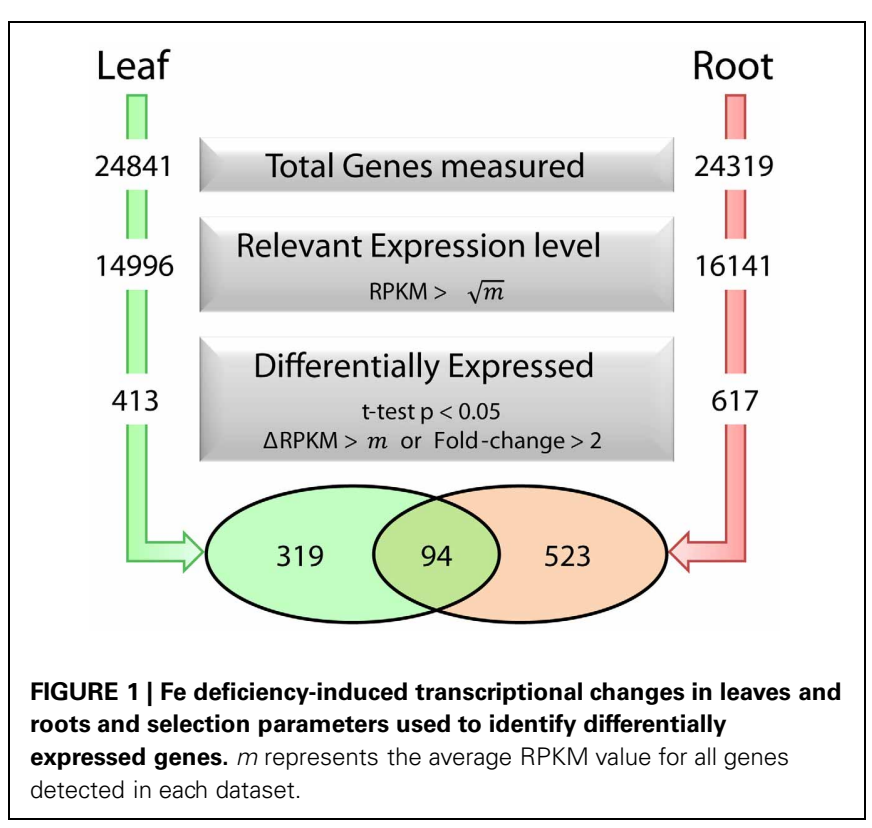

subunits PSAF and PSAN and of the ferredoxin FED2 was decreased by more than 1000 RPKM; transcript levels of several other photosynthesis-associated genes such as the light harvesting complexes-related proteins LHCB6, LHCA3, LHCA2, and LHCB4.1 were reduced by ca. 500 RPKM when grown on media lacking Fe. Furthermore, genes encoding Chl-binding proteins were strongly down-regulated. Interestingly, a subset of seven unknown proteins (At1g47400, At2g14247, At1g13609, At1g47395, At3g56360, At2g30766, and At5g67370) was most strongly up-regulated in leaves. In addition, the protein kinase ORG1, the oligopeptide transporter OPT3 and the Ib subgroup transcription factors $b H L H 38, b H L H 39, b H L H 100$, and $b H L H 101$ were strongly induced by Fe deficiency.

\section{CHLOROPHYLL SYNTHESIS, CHL $b$ TO CHL a CONVERSION AND XANTHOPHYLL SYNTHESIS COMPRISE A “PHOTOOXIDATIVE DAMAGE AVOIDANCE" MODULE}

The universal symptom of $\mathrm{Fe}$-deficient plants is interveinal chlorosis of developing leaves, which is the cause of the decreased Chl content. We found that the Glu-tRNA reductase (HEMA1), which catalizes the reduction of glutamyl-tRNA to glutamate-1semialdehyde, the first committed step in tetrapyrrole biosynthesis, was massively down-regulated in Fe-deficient plants (Figure 2). The product of this first subdivision of Chl synthesis is 5-aminolevulinic acid (ALA). Down-regulation of HEMA1 was robust and occurred rapidly after exposure of the plants to media lacking Fe. We observed a 2-fold decrease in transcript abundance in leaves of hydroponically grown plants after transfer of the plants to Fe-free media within $2 \mathrm{~h}$ (Figure 2, inset), suggesting a prompt adjustment of ALA biosynthesis to the available Fe. The second subdivision of the Chl biosynthesis pathway comprises six steps common to the heme, siroheme and Chl branches in which ALA is converted to protoporphyrin IX (Proto IX). None of the genes encoding enzymes mediating these common steps in tetrapyrrole biosynthesis was affected by Fe deficiency at the transcriptional level. Strong down-regulation occurred in three genes in the Chl branch, namely GUN5, PORB, and CHLP (Figure 2). This branch starts with the insertion of $\mathrm{Mg}^{2+}$ into Proto IX, mediated by magnesium chelatase (GUN5). PORB is one out of three Arabidopsis protochlorophyllide (Pchlide) oxidoreductases that catalize the reduction of the $\mathrm{C} 17-\mathrm{C} 18$ double bond in the $\mathrm{D}$ pyrrole ring to yield chlorophyllide $a$. CHLP encodes a geranylgeranyl reductase that catalyzes the reduction of geranylgeranyl pyrophosphate to phytyl pyrophosphate (Figure 2). A marked decrease in expression was also observed for $N O N$ YELLOW COLORING1 (NYC1), mediating the conversion of Chl $b$ to $\mathrm{Chl} a$. This conversion is important for Chl breakdown and is critical in light regulation (Tanaka et al., 2011). It can be assumed that the rate of Chl breakdown is adjusted to the decreased $\mathrm{Chl}$ synthesis under Fe-deficient conditions. Enzymes of the heme branch were not transcriptionally regulated by Fe. This is contrary to the anticipation since a lack of Fe will compromise the activity of ferrochelatases, inserting $\mathrm{Fe}^{2+}$ into proto IX to form protoheme. A weak down-regulation was observed for UPM1, catalyzing two steps in the siroheme branch.

CONSERVED IN THE GREEN LINEAGE AND DIATOMS27 (CGLD27) belongs to a group of strongly up-regulated leaf 


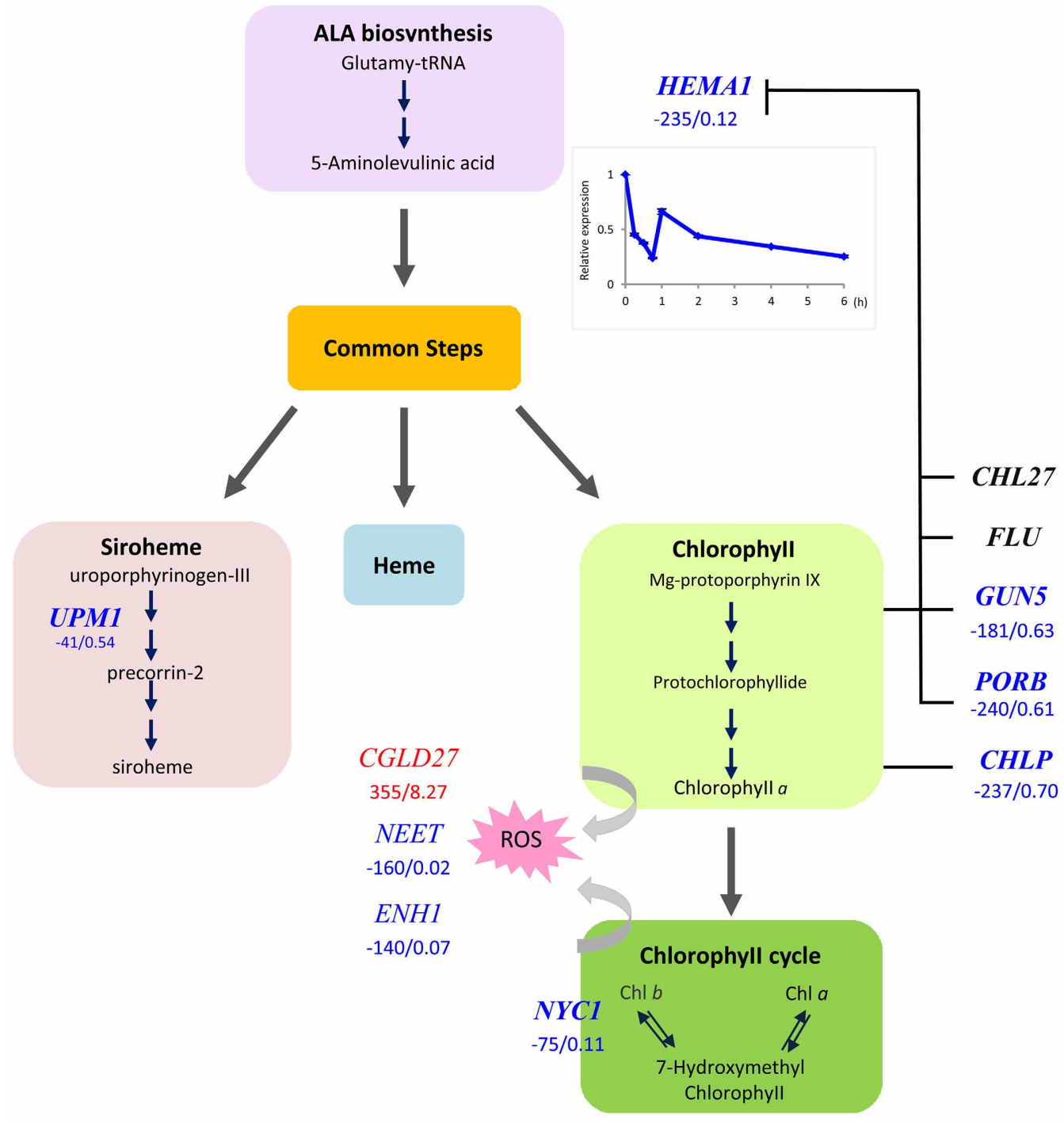

FIGURE 2 | Flow chart of the tetrapyrrole pathway toward siroheme, heme, and chlorophyll. Enzymes that were transcriptionally affected by Fe deficiency are indicated by red (up-regulated genes) or blue letters (down-regulated genes). Numbers indicate delta RPKM and fold change of samples from Fe deficient compared with Fe-sufficient plants. Arrows denote steps that are likely regulated by the [Fe]. Inset shows the time-course of HEMA1 expression in leaves after transfer to Fe-deplete media. genes. CGLD27 has been described as one out of $14 \mathrm{Fe}-$ responsive orthologs in Chlamydomonas and Arabidopsis, indicating that CGLD27 is a common and potentially important component of the Fe deficiency response of the plant lineage (Urzica et al., 2012). CGLD27 is conserved in cyanobacteria and plastid-containing organisms but not in non-photosynthetic organisms, and CGLD27 likely targeted to plastids. Homozygous mutants are more sensitive to low Fe concentrations in the media (Urzica et al., 2012). The protein was predicted to function in carotenoid-xanthophyll metabolism (Kourmpetis et al., 2010) and is co-expressed with the ZETA-CAROTENE DESATURASE (ZDS; At3g04870) involved in the biosynthesis of carotenes and xanthophylls. It is tempting to speculate that CGLD27 has a function in photoprotection by quenching free radicals and singlet oxygen. Together the results indicate that HEMA1, NYC1, and
CGLD27 may represent key players in preventing photooxidative damage in Fe-deficient leaf cells.

Two other genes encoding proteins that are targeted to chloroplast, AtNEET and NAP1 were strongly down-regulated in leaves from Fe-deficient plants. For both genes ancient roles in $\mathrm{Fe}$ metabolism were proposed ( $\mathrm{Xu}$ et al., 2005; Nechushtai et al., 2012), indicating that these proteins may play a role in coordinating tetrapyrrole synthesis and ROS detoxification with the amount of available Fe.

\section{CO-EXPRESSION NETWORKS FROM ROOTS AND SHOOTS ALLOWS AN INTEGRATIVE VIEW OF THE ARABIDOPSIS FE DEFICIENCY RESPONSE}

Co-expression networks can group genes to functionally related modules and can assign putative functions to unknown proteins. To identify modules with functions in the leaf response to $\mathrm{Fe}$ 
deficiency, we subjected the differentially expressed genes identified in our data set to a co-expression analysis using the MACCU software package (Lin et al., 2011). In an attempt to identify conserved and potentially co-regulated processes common to both leaves and roots, we compared transcriptional changes induced by growth on Fe depleted media in leaves with those of roots by re-analyzing a previously published data set of roots from plants grown under the same conditions (RodríguezCelma et al., 2013). In roots from Fe-deficient plants, 617 genes were found to be differentially expressed when similar criteria were applied (Figure 1). We then analyzed the co-expression of genes in leaves and roots separately using databases of publically available microarray experiments that discriminate for leaf- and root-related processes. Finally, we merged the two sub-networks, yielding a large hybrid network comprising 725 genes that can be subdivided in several subclusters (Figure 3, Table S1 and Figure S1). Two subclusters (Hub_1 and Hub_2) connect the two major clusters. The cluster Root_C2 contains genes involved in the acquisition of $\mathrm{Fe}$ and in regulation of $\mathrm{Fe}$ homeostasis. The largest changes in transcript abundance were observed for

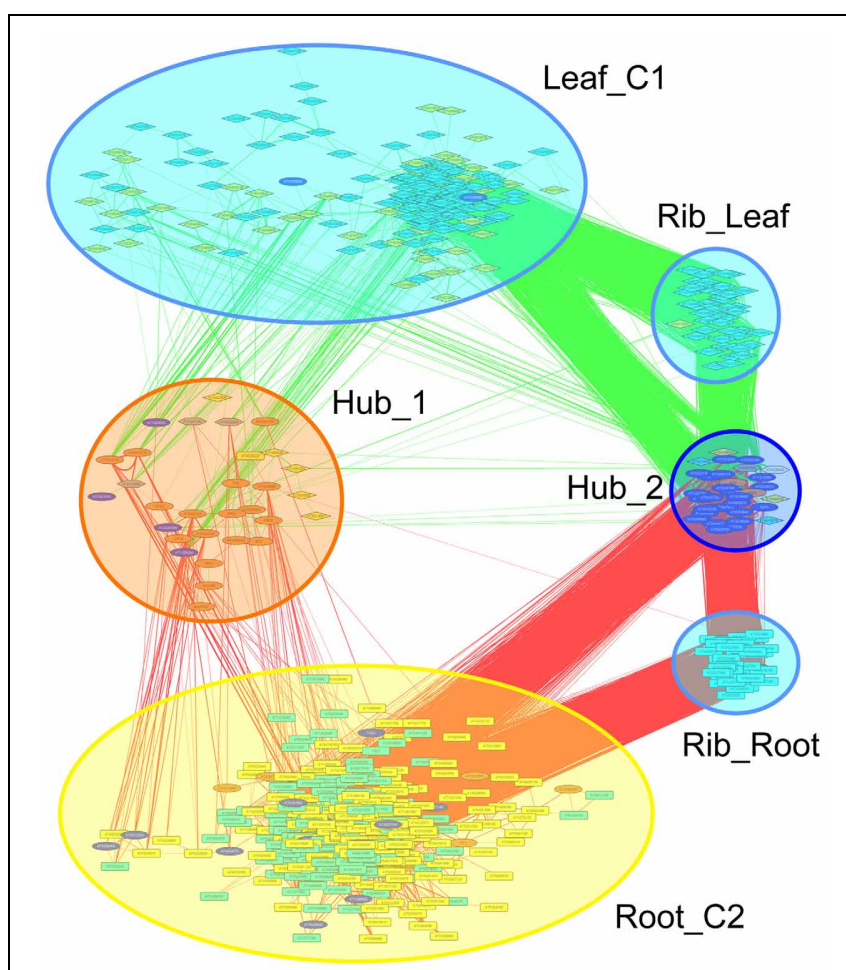

FIGURE 3 | Merged co-expression network of differentially expressed genes in roots and leaves. Differentially expressed genes were first clustered separately for roots and shoots based on their co-expression relationships with a Pearson's coefficient of $\geq 0.60$ against a customized data base that was constructed from experiments conducted with roots or leaves, respectively, using the MACCU software package, and then the two networks were combined. Yellow nodes denote genes that were up-regulated in roots or shoots, orange nodes indicate genes with increased expression in both organs. Light blue nodes denote genes that were down-regulated in roots or shoots, dark blue nodes indicate genes with decreased expression in both organs. Red and green lines represent co-expression edges derived from the root or leaf networks, respectively. genes that mediate the acquisition of Fe (IRT1 and AHA2), genes catalyzing the first steps in the general phenylpropanoid pathway (F6'H1, PAL1, PAL2), the detoxification of transition metals (MTPA2) and in the regulation of Fe homeostasis (FIT). Also, several genes with yet undefined function in Fe homeostasis were highly responsive to the change in growth regime. The most strongly down-regulated gene was PYK10, encoding a $\beta$-glucosidase located in the ER (Nitz et al., 2001). PYK10 was suggested to restrict colonization of the beneficial endophytic fungus Piriformospora indica, thereby conferring resistance to heavy metal ions and promoting nutrient uptake (Sherameti et al., 2008). It can be assumed that lowered expression of PYK10 compensates for reduced interaction between roots and the fungus due to decreased Fe content of root cells. While this assumption awaits further experimental support, the data might hint at an understudied aspect of the Fe-deficiency response that may be of importance for the fitness of plants under natural conditions.

The cluster Leaf_C1 contained several genes associated with PS, LHCs and with Chl synthesis that have been described above. Genes derived from the root and shoot network were connected by three relatively large subclusters containing ribosome genes. Notably, two of the ribosomal subclusters (Rib_Leaf and Rib_Root) were comprised of genes that were exclusively derived from the root and shoot network, and the third subcluster contained genes from both subnetworks. Inspection of the genes in these subclusters revealed that the down-regulation of genes encoding ribosomal proteins was differential between leaves or roots. Notably, in leaves proteins belonging to paralogs families of the large subunit were affected by Fe deficiency, while in roots the majority of regulated ribosomal genes encoded ribosomal proteins from the $40 \mathrm{~S}$ subunit (Table S2). This suggests that the composition of ribosomes is changed by Fe deficiency and that this change occurs in an organ-specific manner (Figure 4).

\section{A CONSERVED SET OF FE-REGULATED GENES IS HIGHLY EXPRESSED IN BOTH LEAVES AND ROOTS}

Using conservative criteria ( $\mathrm{FC}>2, P<0.05$ and $\triangle \mathrm{RPKM}>40$ ), we mined the data sets for genes that are strongly Fe-regulated in both leaves and roots. A set of 17 genes fulfilled these criteria, among them the transcription factors $b H L H 38, b H L H 39$ and bHLH100, FRO3, OPT3, the nicotianamine synthase NAS4, the Fe storage protein FER1, the Fe superoxide dismutase FDS1 and the protein kinase ORG1 (Table 1), some of which were reported to be $\mathrm{Fe}$-regulated in previous microarray experiments (Ivanov et al., 2011). Furthermore, six proteins in this set have unknown functions (Table 1). The high expression levels and the robust regulation of the genes encoding these proteins indicate putatively important functions in Fe metabolism. We have named them IRON-RESPONSIVE PROTEIN (IRP) 1-6, as candidates for follow-up experiments (Table 1). The encoded proteins can be organized in three groups of two proteins. IRP1 (At1g47400) and IRP2 (Atlg47395) are peptides of 50 amino acids with a conserved region. The second group comprises IRP3 (At2g14247) and IRP4 (At1g13609), small proteins of 78 amino acids and no conserved domain. IRP5 (At3g56360) and IRP6 (At5g05250) are 25.1 and $25.9 \mathrm{kDa}$ proteins, respectively, with predicted myristoylation sites. 


\section{Root Ribosome}

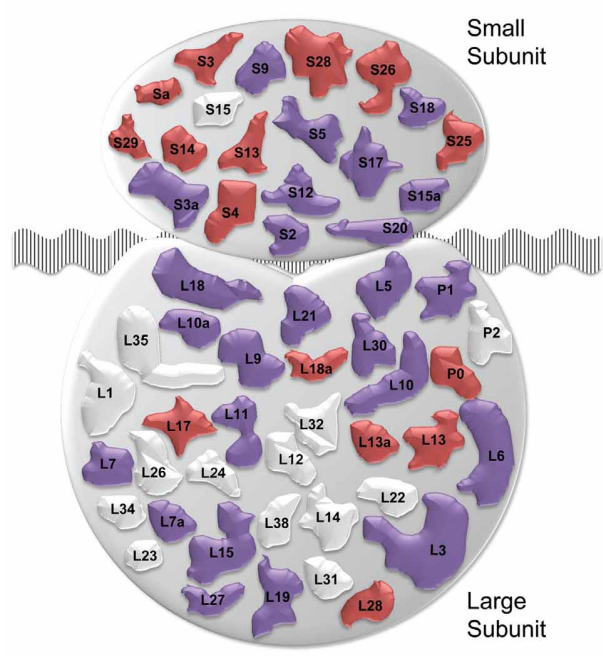

FIGURE 4 | Fe deficiency-induced changes in ribosomal composition. Schematic of ribosomal composition changes for roots and leaves. Red and green shapes represent ribosomal protein families down-regulated

\section{Leaf Ribosome}

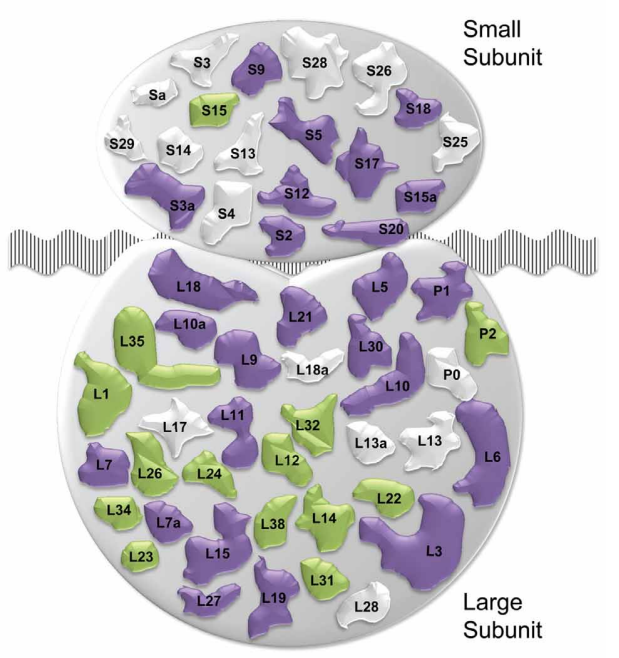

specifically in roots and leaves, respectively. Purple colored shapes represent families down-regulated in both organs. White colored shapes denote families not regulated in the corresponding tissue ribosomes.

Table 1 | Genes that were differentially expressed between Fe-sufficient and Fe-deficient plants in both leaves and roots with a $\triangle R P K M>40$ and $\mathrm{FC}>2$ at $P<0.05$.

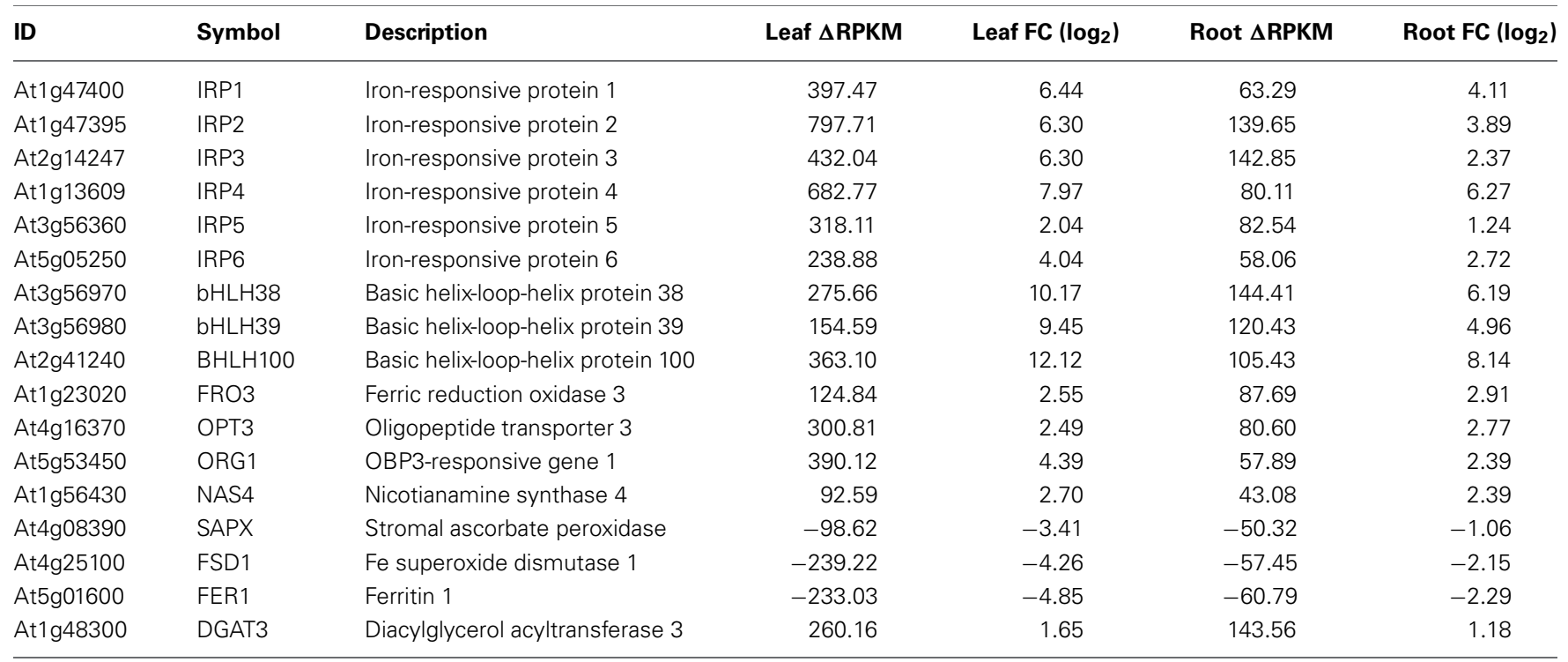

To put the conserved genes into a functional context and to assign putative functions for the unknown proteins, we constructed a co-expression network using the set of 17 conserved genes as baits and fished for first degree co-expressed nodes (Figure 5). The resulting network was comprised 99 genes with several central players in Fe homeostasis such as OPT3, FRO3, NAS4, IRT1, MTPA2, and the phosphoenolpyruvate carboxylase PPC1. Also, four major regulators of Fe homeostasis were found within this network, including BTS, PYE, bHLH39, and bHLH101. Notably, with the exception of IRT1, MTPA2, and $P P C 1$, which appear more important for root Fe acquisition, all the genes listed above plus HEMA1 and FER4 were located in HUB_1 (Figure 3) that connects the genes that are specific for roots or leaves, indicating a function of these genes in both organs and/or in systemic Fe homeostatic regulation. This network also contained 16 genes encoding ribosomal proteins and two orthologs of the mammalian receptor for activated C-kinase 1 (RACK1), RACK1A, and RACK1B. In both mammals and plants, RACK1 genes have been associated with protein translation and ribosome biogenesis (Nilsson et al., 2004; Guo et al., 2011). Together these results indicate conserved players in cellular $\mathrm{Fe}$ homeostasis. They establish a link between several novel genes 


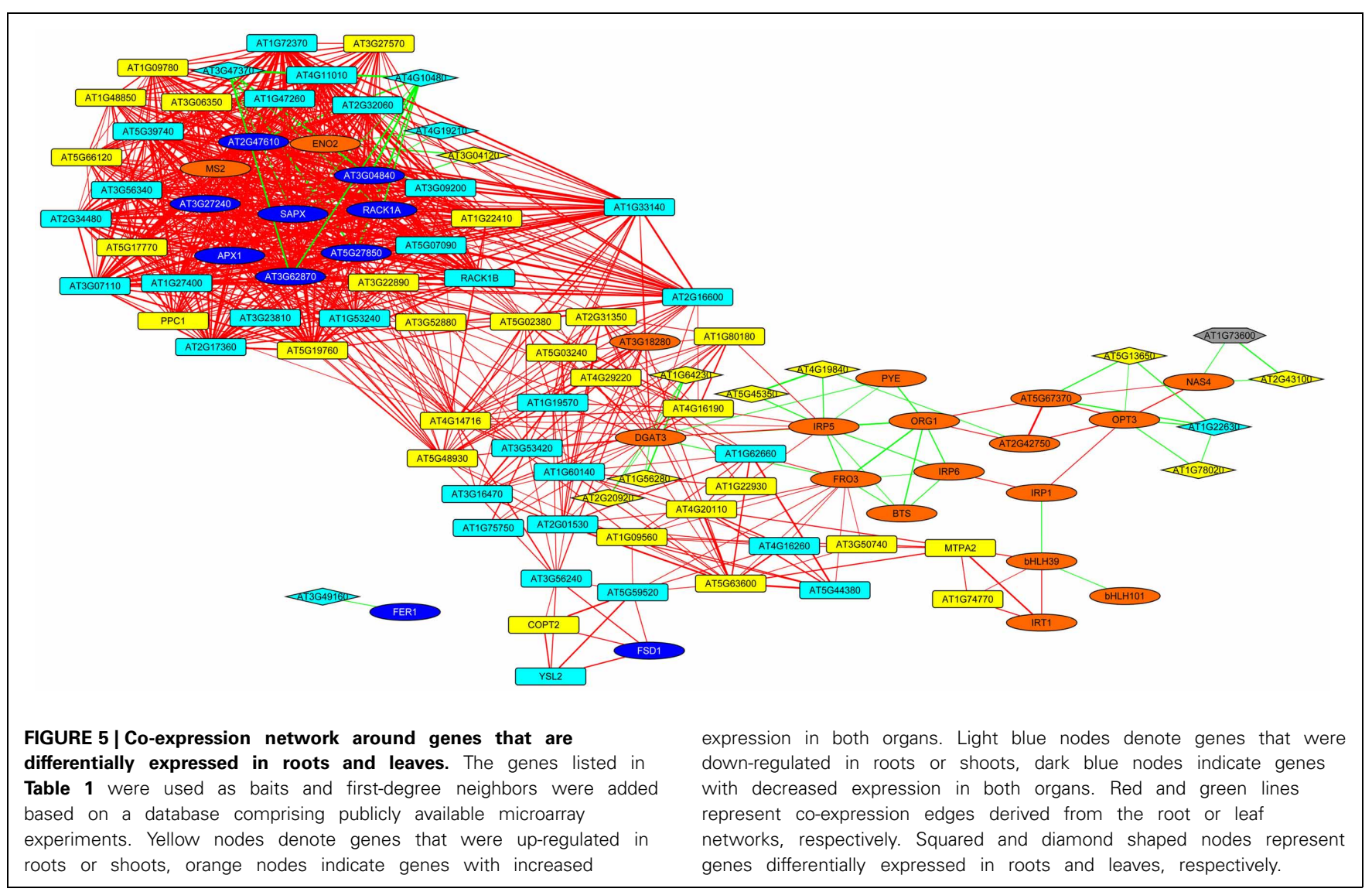

encoding proteins with unknown functions and a regulatory network.

\section{DISCUSSION}

While the transcriptional response of roots to Fe deficiency is well explored at the whole-genome level, information on transcriptional profile changes that occur in Fe-deficient leaves is scarce. The largest sinks for Fe are PSI and PSII, and the major fraction of $\mathrm{Fe}$ is located in the chloroplasts. It seems reasonable to assume that photosynthetic cells have evolved mechanisms that adjust the synthesis of light harvesting complexes to that of Fe-containing enzymes and complexes to avoid accumulation of ROS and to recalibrate Fe homeostasis in leaf cells.

\section{PHOTOOXIDATIVE DAMAGE IN FE-DEFICIENT LEAF CELLS IS AVOIDED BY ELABORATE MECHANISMS}

Decreased Chl content is a hallmark of Fe-deficient plants and has been associated with compromised photosynthesis caused by decreased tetrapyrrole biosynthesis. The fast and pronounced down-regulation of HEMA1 expression suggests a rapid and dramatic decline of tetrapyrrole synthesis at an early stage of $\mathrm{Fe}$ deficiency. Our data support a scenario in which plastid Fe sensing links Chl synthesis to the activity of the photosystems to avoid the production of toxic radicals upon illumination of free tetrapyrrole intermediates. Notably, Fe-deficient Clamydomonas cells are not sensitive to high light and photosynthetic mutants grow better on low Fe media (Moseley et al., 2002), demonstrating that Fe-deficient plants are protected against photo-oxidative damage. ALA synthesis is rate-limiting in tetrapyrrole synthesis and sophisticatedly regulated (Tanaka et al., 2011; Brzezowski and Grimm, 2013). Interestingly, in acetate-grown Chlamydomonas cells tetrapyrrole synthesis was up-regulated upon Fe deficiency, while photoautotrophic cells showed lower mRNA levels of the enzymes involved in the pathway (Moseley et al., 2002), suggesting that Chl synthesis is not restricted by decreased Fe supply. Similar to other regulators of tetrapyrrole synthesis such as light, phytohormones and the circadian clock, the control sites with regard to $\mathrm{Fe}$ availability are at the beginning, at the branch points and at the end of the pathway. This avoids the accumulation of photoreactive porphyrin and of free $\mathrm{Chl}$ intermediates, and adjusts the metabolic flow to demand. The metabolites $\mathrm{Mg}$ protoporphyrin and divinylprotochlorophylide $a$ negatively regulate HEMA1 activity (Tanaka and Tanaka, 2007). Recently, it has been shown that in the presence of Pchlide a complex comprising the fluorescence protein FLU, PORB, CHL27, and CHLP interacts with HEMA1 and inhibits ALA synthesis (Figure 2; Kauss et al., 2012). In the absence of Pchlide, such interaction is compromised and allows ALA synthesis (Kauss et al., 2012). How are Chl synthesis and photosynthetic activity synchronized to avoid accumulation of ROS in Fe-deficient leaves? A possible scenario involves a Chl-mediated disconnection of the $\mathrm{LHCl}$ antenna from PSI as described for Chlamydomonas (Moseley et al., 2002). A finely tuned Chl synthesis and binding of Chl to PSI would allow an efficient adaptation of the photosynthetic machinery 
to the available Fe pools. The diiron Mg-protoporphyrin IX monomethylester oxidative cyclase CHL27 has been suggested as a candidate plastid Fe sensor (Tottey et al., 2003). Another potential candidate that links photosynthesis, ROS and Fe metabolism is the NEET protein At5g51720 (AtNEET). AtNEET harbors two redox active $2 \mathrm{Fe}-2 \mathrm{~S}$ clusters and is dramatically downregulated (40-fold; 98\% decrease in transcript level) in leaves of Fe-deficient plants. AtNEET is tightly co-expressed with FER3 and FER4, encoding plastid ferritins. Furthermore, AtNEET is co-expressed with ENH1, which may play a role in ROS detoxification (Zhu et al., 2007). Reduced levels of AtNEET transcript levels caused late greening, increased the sensitivity to low Fe levels, and conferred insensitivity to high Fe concentrations (Nechushtai et al., 2012). NEET proteins are conserved in plants and mammals and an ancient role of AtNEET in Fe homeostasis has been suggested (Nechushtai et al., 2012). However, a clear role in plastid Fe metabolism has not yet been defined. Another candidate for a plastid Fe sensor is the NONINTRINSIC ABC PROTEIN (NAP1), a protein with similarities to prokaryotic $S u f B$ proteins which are involved in Fe-S cluster repair (Xu et al., 2005). AtNAP1 complements an E. coli SufB mutant, indicating that AtNAP1 is an evolutionary conserved SufB protein (Xu et al., 2005), and regulation of cellular sulfur level has been previously shown to be related to Fe homeostasis (Ivanov et al., 2011). AtNAP1 is transcriptionally and post-transcrioptionally regulated by $\mathrm{Fe}$, and may function in plastid Fe homeostasis (Xu et al., 2005). A strong down-regulation of AtNAP1 was observed in the present study (Table S1), supporting the assumption of a role of AtNAP1 in plastid Fe sensing or signaling.

\section{FE DEFICIENCY ALTERS RIBOSOME COMPOSITION IN AN ORGAN-SPECIFIC MANNER}

While considered as housekeeping genes encoding structural components, plant cytosolic ribosomes are more heterogenous and plastic than their mammalian counterparts. Plant ribosomal proteins are encoded by multiple divergent paralogs, which allows, in theory, for $10^{34}$ structurally different ribosomes (Hummel et al., 2012), a number that can be increased by numerous post-translational modifications. Generally, ribosome biogenesis appears to be down-regulated in both roots and leaves from Fe-deficient plants. A large part of transcriptional activity is used for this process and the decrease in the abundance of ribosomal subunits may be interpreted in terms of biasing translation toward proteins encoding products that are needed in large amounts to recalibrate cellular Fe homeostasis. It also appears, however, that the down-regulation is not general but highly organ-specific, indicating a carefully controlled change in ribosomal composition. Paralog composition has also been shown to be altered by growth conditions (Hummel et al., 2012), which is most likely the case in Fe-deficient plants. The fact that the change in the expression of paralogous isoforms is typical of leaves and roots suggests that this change is not due to a general down-regulation of translation, but rather may adjust the protein composition of the cell to the prevailing conditions. This would also offer an explanation for the relatively low concordance of proteomic and transcriptomic changes in response to environmental conditions (Lan et al., 2012). Importantly, our results indicate that translational bias, mediated by a change in composition of ribosomes, is part of the Fe deficiency response of both leaves and roots.

\section{ESTABLISHED AND NOVEL PLAYERS CONTROL CELLULAR FE HOMEOSTASIS IN LEAVES AND ROOTS}

Despite the different function of leaf and root cells, many of the major regulators of Fe deficiency responses that have been identified in roots were also found in leaves, indicating that the subgroup $1 \mathrm{~b}$ transcription factors and PYE/BTS are controlling cellular Fe homeostasis in most if not all plant cells (Ivanov et al., 2011). A notable exception was FIT, the expression of which appears to be root-specific. It seems plausible that different combinations of heterodimers are regulating non-overlapping sets of genes, a scenario which does not exclude other levels of regulation. A novel finding was the differential expression of ribosomal proteins, which possibly altered the composition of ribosomes in an organ and growth type-specific manner, a regulatory layer which has yet to be explored. Our data also revealed several new players that may play conserved roles in Fe metabolism in both leaves and roots. Some of these genes have no corresponding probe set on the ATH1 gene chip and can thus not be properly inserted into co-expression networks. The three genes for which a co-expression relationship can be inferred from public data bases (IRP1, IRP5, and IRP6), are closely co-expressed with each other and several genes with important functions in Fe homeostasis (e.g., NRAMP4, FRO3, NAS4, bHLH39, OPT3, ORG1, and PYE), associating putative roles in Fe metabolism of these proteins in both leaves and roots.

No transporter from the YLS, ZIP, or IREG family that could mediate the transport of $\mathrm{Fe}$ (II) has been found to be Fe-regulated in leaf cells. Such a function could be fulfilled by OPT3, as inferred from the strong phenotype of the opt3-2 mutant (Stacey et al., 2008), the general characteristics and expression patterns of OPT transporters (Lubkowitz, 2011), the massive up-regulation of the genes upon Fe deficiency and the central position in the co-expression networks.

Several lines of evidence support a concept of anticipated changes that have been proven for the sequestering of surplus metals that are associated with an up-regulation of IRT1 activity (Yang et al., 2010). For example, down-regulation of PYK10 can be interpreted as an anticipation of a Fe deficiency-induced decrease in colonization efficiency of $P$. indica. Also, the fast orchestrated regulation of tetrapyrrole synthesis, $\mathrm{Chl} b$ to a $\mathrm{Chl}$ $a$ conversion and, putatively, the biosynthesis of xanthophyll to avoid oxidative damage may be interpreted as anticipatory changes. Understanding of the regulation of these response modules is desirable in order to gain a holistic understanding of the responses to Fe deficiency and also in order to develop tools to generate plants with improved Fe efficiency.

\section{ACKNOWLEDGMENTS}

This work was supported by grants from Academia Sinica.

\section{SUPPLEMENTARY MATERIAL}

The Supplementary Material for this article can be found online at http://www.frontiersin.org/Plant_Nutrition/10.3389/fpls.2013. 00276/abstract 


\section{REFERENCES}

Brzezowski, P., and Grimm, B. (2013). Chlorophyll metabolism. eLS. doi: 10.1002/9780470015902.a0020084. pub2

Buckhout, T. J., Yang, T. J. W., and Schmidt, W. (2009). Early irondeficiency-induced transcriptional changes in Arabidopsis roots as revealed by microarray analyses. BMC Genomics 10:147. doi: 10.1186/1471-2164-10-147

Colangelo, E. P., and Guerinot, M. L. (2004). The essential basic helixloop-helix protein FIT1 is required for the iron deficiency response. Plant Cell 16, 3400-3412. doi: 10.1105/tpc. 104.024315

Didonato, R. J., Jr., Roberts, L. A., Sanderson, T., Eisley, R. B., and Walker, E. L. (2004). Arabidopsis Yellow Stripe-Like2 (YSL2): a metal-regulated gene encoding a plasma membrane transporter of nicotianaminemetal complexes. Plant J. 39, 403-414. doi: 10.1111/j.1365-313X. 2004.02128.x

Eide, D., Broderius, M., Fett, J., and Guerinot, M. L. (1996). A novel iron-regulated metal transporter from plants identified by functional expression in yeast. Proc. Natl. Acad. Sci. U.S.A. 93, 5624-5628. doi: 10.1073/pnas.93.11.5624

Estelle, M. A., and Somerville, C. (1987). Auxin-resistant mutants of Arabidopsis thaliana with an altered morphology. Mol. Gen. Genet. 206, 200-206. doi: 10.1007/BF003 33575

Gris, E. (1844). Nouvelles expériences sur l'action des composés ferrugineux solubles, et de la debilite des plantes. CR Acad. Sci. Paris 19, 1118-1119.

Guo, J., Wang, S., Valerius, O., Hall, H., Zeng, Q., Li, J. F., et al. (2011). Involvement of Arabidopsis RACK1 in protein translation and its regulation by abscisic acid. Plant Physiol. 155, 370-383. doi: 10.1104/pp.110.160663

Hummel, M., Cordewener, J. H. G., De Groot, J. C. M., Smeekens, S., America, A. H. P., and Hanson, J. (2012). Dynamic protein composition of Arabidopsis thaliana cytosolic ribosomes in response to sucrose feeding as revealed by label free MSE proteomics. Proteomics 12, 1024-1038. doi: 10.1002/pmic.201100413

Ivanov, R., Brumbarova, T., and Bauer, P. (2011). Fitting into the harsh reality: regulation of iron-deficiency responses in dicotyledonous plants. Mol. Plant 5, 27-42. doi: $10.1093 / \mathrm{mp} / \mathrm{ssr} 065$
Jakoby, M., Wang, H. Y., Reidt, W., Weisshaar, B., and Bauer, P. (2004). FRU (BHLH029) is required for induction of iron mobilization genes in Arabidopsis thaliana. FEBS Lett. 577, 528-534. doi: 10.1016/j.febslet. 2004.10.062

Kauss, D., Bischof, S., Steiner, S., Apel, K., and Meskauskiene, R. (2012). FLU, a negative feedback regulator of tetrapyrrole biosynthesis, is physically linked to the final steps of the $\mathrm{Mg}(++)$-branch of this pathway. FEBS Lett. 586, 211-216. doi: 10.1016/j.febslet. 2011.12.029

Kent, W. J. (2002). BLAT - the BLASTlike alignment tool. Genome Res. 12, 656-664. doi: 10.1101/gr.229202

Kourmpetis, Y. A., Van Dijk, A. D., Bink, M. C., Van Ham, R. C., and TerBraak, C. J. (2010). Bayesian Markov Random Field analysis for protein function prediction based on network data. PLOS ONE 5:e9293. doi: 10.1371/journal.pone. 0009293

Lan, P., Li, W., and Schmidt, W. (2012). Complementary proteome and transcriptome profiling in phosphate-deficient Arabidopsis roots reveals multiple levels of gene regulation. Mol. Cell. Proteomics 11, 1156-1166. doi: 10.1074/mcp.M112.020461

Lin, W. D., Liao, Y. Y., Yang, T. J., Pan, C. Y., Buckhout, T. J., and Schmidt, W. (2011). Coexpressionbased clustering of Arabidopsis root genes predicts functional modules in early phosphate deficiency signaling. Plant Physiol. 155, 1383-1402. doi: 10.1104/pp.110. 166520

Ling, H. Q., Bauer, P., Bereczky, Z., Keller, B., and Ganal, M. (2002). The tomato fer gene encoding a bHLH protein controls iron-uptake responses in roots. Proc. Natl. Acad. Sci. U.S.A. 99, 13938-13943. doi: $10.1073 /$ pnas. 212448699

Long, T. A., Tsukagoshi, H., Busch, W., Lahner, B., Salt, D. E., and Benfey, P. N. (2010). The bHLH transcription factor POPEYE regulates response to iron deficiency in Arabidopsis roots. Plant Cell 22, 2219-2236. doi: 10.1105/tpc.110.07 4096

Lubkowitz, M. (2011). The oligopeptide transporters: a small gene family with a diverse group of substrates and functions? Mol . Plant 4, 407-415. doi:10.1093/ $\mathrm{mp} / \mathrm{ssr} 004$

Mortazavi, A., Williams, B. A., McCue, K., Schaeffer, L., and Wold, B. (2008). Mapping and quantifying mammalian transcriptomes by RNA-Seq. Nat. Methods 5, 621-628. doi: 10.1038/nmeth. 1226

Moseley, J. L., Allinger, T., Herzog, S., Hoerth, P., Wehinger, E., Merchant, S., et al. (2002). Adaptation to Fe-deficiency requires remodeling of the photosynthetic apparatus. EMBO J. 21, 6709-6720. doi: 10.1093/emboj/cdf666

Nechushtai, R., Conlan, A. R., Harir, Y., Song, L., Yogev, O., EisenbergDomovich, Y., et al. (2012). Characterization of Arabidopsis NEET reveals an ancient role for NEET proteins in iron metabolism. Plant Cell 24, 2139-2154. doi: 10.1105/tpc.112.097634

Nilsson, J., Sengupta, J., Frank, J., and Nissen, P. (2004). Regulation of eukaryotic translation by the RACK1 protein: a platform for signalling molecules on the ribosome. EMBO Rep. 5, 1137-1141. doi: 10.1038/sj.embor. 7400291

Nitz, I., Berkefeld, H., Puzio, P. S., and Grundler, F. M. (2001). PYK10, a seedling and root specific gene and promoter from Arabidopsis thaliana. Plant Sci. 161, 337-346. doi: 10.1016/S01689452(01)00412-5

op den Camp, R. G., Przybyla, D. Ochsenbein, C., Laloi, C., Kim C., Danon, A., et al. (2003). Rapid induction of distinct stress responses after the release of singlet oxygen in Arabidopsis. Plant Cell 15, 2320-2332. doi: $10.1105 /$ tpc. 014662

Rellán-Álvarez, R., Giner-MartínezSierra, J., Orduna, J., Orera, I., Rodríguez-Castrillón, J. A., García-Alonso, J. I., et al. (2010). Identification of a tri-iron(III), tri-citrate complex in the xylem sap of iron-deficient tomato resupplied with iron: new insights into plant iron long-distance transport. Plant Cell Physiol. 51, 91-102. doi: 10.1093/pcp/pcp 170

Robinson, N. J., Procter, C. M., Connolly, E. L., and Guerinot, M. L. (1999). A ferric-chelate reductase for iron uptake from soils. Nature 397, 694-697.

Rodríguez-Celma, J., Lin, W.-D., Fu, G.-M., Abadia, J., López-Millán, A.-F., and Schmidt, W. (2013). Mutually exclusive alterations in secondary metabolism are critical for the uptake of insoluble iron compounds by Arabidopsis and Medicago truncatula. Plant Physiol. 162, 1473-1485. doi: 10.1104/pp. 113.220426

Santi, S., and Schmidt, W. (2009). Dissecting iron deficiency-induced proton extrusion in Arabidopsis roots. New Phytol. 183, 1072-1084. doi: $\quad 10.1111 /$ j.1469-8137.2009. 02908.x

Sherameti, I., Venus, Y., Drzewiecki, C., Tripathi, S., Dan, V. M., Nitz, I., et al. (2008). PYK10, a betaglucosidase located in the endoplasmatic reticulum, is crucial for the beneficial interaction between Arabidopsis thaliana and the endophytic fungus Piriformospora indica. Plant J. 54, 428-439. doi: 10.1111/ j.1365-313X.2008.03424.x

Stacey, M. G., Patel, A., McClain, W. E., Mathieu, M., Remley, M., Rogers, E. E., et al. (2008). The Arabidopsis AtOPT3 protein functions in metal homeostasis and movement of iron to developing seeds. Plant Physiol. 146, 589-601. doi: 10.1104/pp.107. 108183

Tanaka, R., Kobayashi, K., and Masuda, T. (2011). Tetrapyrrole metabolism in Arabidopsis thaliana. Arabidopsis Book 9:e0145. doi: 10.1199/ tab.0145

Tanaka, R., and Tanaka, A. (2007). Tetrapyrrole biosynthesis in higher plants. Annu. Rev. Plant Biol. 58, 321-346. doi: 10.1146/annurev. arplant.57.032905.105448

Terry, N. (1980). Limiting factors in photosynthesis: I. Use of iron stress to control photochemical capacity in vivo. Plant Physiol. 65, 114-120. doi: 10.1104/pp.65.1.114

Tottey, S., Block, M. A., Allen, M., Westergren, T., Albrieux, C., Scheller, H. V., et al. (2003). Arabidopsis CHL27, located in both envelope and thylakoid membranes, is required for the synthesis of protochlorophyllide. Proc. Natl. Acad. Sci. U.S.A. 100, 16119-16124. doi: 10.1073/pnas.2136793100

Urzica, E. I., Casero, D., Yamasaki, H., Hsieh, S. I., Adler, L. N., Karpowicz, S. J., et al. (2012). Systems and trans-system level analysis identifies conserved iron deficiency responses in the plant lineage. Plant Cell 24, 3921-3948. doi: 10.1105/tpc.112.102491

Wang, L., Cui, Y., Liu, Y., Fan, H., Du, J., Huang, Z., et al. (2012). Requirement and Functional Redundancy of Ib subgroup bHLH proteins for iron deficiency responses and uptake in Arabidopsis thaliana. Mol. Plant 6, 503-513. doi: 10.1093/mp/sss089

Waters, B. M., Chu, H. H., Didonato, R. J., Roberts, L. A., Eisley, R. B., Lahner, B., et al. (2006). Mutations in Arabidopsis yellow stripe-like1 and yellow stripe-like3 reveal their roles in metal ion homeostasis and loading of metal ions in seeds. 
Plant Physiol. 141, 1446-1458. doi: 10.1104/pp.106.082586

Xu, X. M., Adams, S., Chua, N. H., and Moller, S. G. (2005). AtNAP1 represents an atypical SufB protein in Arabidopsis plastids. J. Biol. Chem. 280, 6648-6654.

Yang, T. J. W., Lin, W. D., and Schmidt, W. (2010). Transcriptional profiling of the Arabidopsis Iron deficiency response reveals conserved transition metal homeostasis networks. Plant Physiol. 152, 2130-2141. doi: 10.1104/pp.109.152728

Yuan, Y., Wu, H., Wang, N., Li, J., Zhao, W., Du, J., et al. (2008). FIT interacts with AtbHLH38 and AtbHLH39 in regulating iron uptake gene expression for iron homeostasis in Arabidopsis. Cell Res. 18, 385-397. doi: 10.1038/cr.2008.26

Yuan, Y. X., Zhang, J., Wang, D. W., and Ling, H. Q. (2005). AtbHLH29 of Arabidopsis thaliana is a functional ortholog of tomato FER involved in controlling iron acquisition in strategy I plants. Cell Res. 15, 613-621. doi: 10.1038/sj.cr.7290331

Zhu, J., Fu, X., Koo, Y. D., Zhu, J. K., Jenney, F. E. Jr., et al. (2007). An enhancer mutant of Arabidopsis salt overly sensitive 3 mediates both ion homeostasis and the oxidative stress response. Mol.
Cell. Biol. 27, 5214-5224. doi: 10.1128/MCB.01989-06

Conflict of Interest Statement: The authors declare that the research was conducted in the absence of any commercial or financial relationships that could be construed as a potential conflict of interest.

Received: 29 May 2013; paper pending published: 19 June 2013; accepted: 04 July 2013; published online: 23 July 2013. Citation: Rodríguez-Celma J, Pan IC, Li W, Lan P, Buckhout TJ and Schmidt $W$ (2013) The transcriptional response of Arabidopsis leaves to Fe deficiency. Front. Plant Sci. 4:276. doi: 10.3389/fpls. 2013.00276

This article was submitted to Frontiers in Plant Nutrition, a specialty of Frontiers in Plant Science.

Copyright (C) 2013 Rodríguez-Celma, Pan, Li, Lan, Buckhout and Schmidt. This is an open-access article distributed under the terms of the Creative Commons Attribution License, which permits use, distribution and reproduction in other forums, provided the original authors and source are credited and subject to any copyright notices concerning any third-party graphics etc. 